\title{
Efecto insecticida de una fracción de acetato de etilo de Serjania schiedeana Schltdl (Sapindaceae) contra Melanaphis sacchari Zehntner (Hemiptera: Aphididae)
}

Insecticidal effect of a fraction of ethyl acetate of Serjania schiedeana Schltdl (Sapindaceae) against Melanaphis sacchari Zehntner (Hemiptera: Aphididae)

\section{David Osvaldo Salinas-Sánchez¹, Guadalupe Peña-Chora², Manasés González-Cortazar³, Alejandro Zamilpa ${ }^{3}$, Víctor Manuel Hernández-Velázquez ${ }^{4}$, Luz Janet Tagle ${ }^{5}$, César Sotelo-Leyva ${ }^{4,5^{*}}$}

\footnotetext{
${ }^{1}$ Centro de Investigación en Biodiversidad y Conservación, Universidad Autónoma del Estado de Morelos (UAEM), Av. Universidad 1001, Col. Chamilpa, 62209, Cuernavaca, Morelos, México. ${ }^{2}$ Centro de Investigaciones Biológicas, UAEM.

${ }^{3}$ Centro de Investigación Biomédica del Sur, Instituto Mexicano del Seguro Social, Calle Rep. Argentina 1, Col. Centro, 62790, Xochitepec, Morelos, México.

${ }^{4}$ Centro de Investigación en Biotecnología, UAEM.

${ }^{5}$ Facultad de Ciencias Químico-Biológicas, Universidad Autónoma de Guerrero, Lázaro Cárdenas s/n, Ciudad Universitaria Sur, 39070 , Chilpancingo de los Bravo, Guerrero, México.

*Autor de correspondencia: cesarsotelo@uagro.mx
}

Fecha de recepción:

5 de febrero de 2020

Fecha de aceptación:

4 de junio de 2020

Disponible en línea:

7 de agosto de 2020

Este es un artículo en acceso abierto que se distribuye de acuerdo a los términos de la licencia Creative Commons.

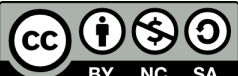

Reconocimiento-

NoComercial-

CompartirIgual 4.0

Internacional

\section{RESUMEN}

El pulgón de la caña de azúcar, Melanaphis sacchari Zehntner (Hemiptera: Aphididae), es una de las principales plagas del sorgo, Sorghum bicolor L. Moench (Poaceae), en México. El manejo actual de las poblaciones del insecto depende del uso de insecticidas sintéticos, pero nuevas alternativas exploran el uso de extractos vegetales. Por lo que el objetivo principal de este estudio fue evaluar el efecto insecticida de contacto de una fracción de acetato de etilo de tallos de Serjania schiedeana (Sapindaceae), contra adultos ápteros de $M$. sacchari en condiciones de laboratorio. Los resultados mostraron que el extracto de S. schiedeana causó una mortalidad de $78 \%$ a 10,000 ppm en 72 h; la mortalidad del control positivo (imidacloprid) fue de 100\% en 24 h, y el control negativo (Tween® 20), de $4 \%$ en 72 h. El análisis de cromatografía de gases-masas (CG-MS) de la fracción de acetato de etilo de S. schiedeana, identificó 12 compuestos, entre los cuales, el mayoritario (38.66\%) fue el palmitato de metilo, un éster de ácido graso común de origen vegetal. La fracción de acetato de etilo de S. schiedeana, presentó una actividad insecticida importante y podría ser considerada como una alternativa para el control de M. sacchari.

PALABRAS CLAVE

Fracción, mortalidad, áfidos, CG-MS, bioactividad, compuestos.

\section{ABSTRACT}

The sugarcane aphid, Melanaphis sacchari Zehntner (Hemiptera: Aphididae), is one of the main insect pests of sorghum, Sorghum bicolor L. Moench (Poaceae), in Mexico. The actual population 
management for this pest is based on synthetic pesticide applications, but new control strategies are focused on plant extracts. For this reason, the main objective of this study was to evaluate the contact insecticidal effect of a fraction of ethyl acetate from stems of Serjania schiedeana (Sapindaceae), against adult apterous M. sacchari under laboratory conditions. The results showed that the $S$. schiedeana extract achieved an aphid mortality of $78 \%$ at 10,000 ppm in $72 \mathrm{~h}$, the positive control (imidacloprid), of $100 \%$ in $24 \mathrm{~h}$, and the negative control (Tween ${ }^{\circledR} 20$ ), of $4 \%$ in $72 \mathrm{~h}$. Gas-mass chromatography (GC-MS) analysis of the ethyl acetate fraction of S. schiedeana identified 12 compounds, from which methyl palmitate, a common plant-derived fatty acid ester, was predominant $(38.66 \%)$. The ethyl acetate fraction of $S$. schiedeana had an important insecticidal activity and could be considered as an alternative for the control of M. sacchari.

\section{keywords}

Fraction, mortality, aphids, CG-MS, bioactivity, compounds.

\section{INTRODUCCIÓN}

El sorgo, Sorghum bicolor L. Moench, es una especie vegetal perteneciente a la familia Poaceae, así como uno de los cultivos más antiguos e históricamente uno de los cereales de mayor importancia mundial (Chuck-Hernández et al. 2011). México ocupa el segundo lugar de producción a nivel mundial, con 6.5 millones de toneladas en el ciclo agrícola 2016/17 (FIRA 2016). Se cultiva en todas las entidades federativas de la República Mexicana, y en Morelos, en el periodo otoño-invierno de 2019 (riego + temporal), se obtuvieron 1,063 ton (SIAP 2019).

El pulgón de la caña de azúcar, Melanaphis sacchari Zehntner (Hemiptera: Aphididae), es de origen africano, e ingresó a Estados Unidos en 1970 como una plaga de la caña de azúcar, Saccharum officinarum L. (Poaceae) (Schenck y Lehrer 2007), pero en 2013 se empezaron a encontrar brotes masivos en cultivos de sorgo en América del Norte, cuyos daños fueron más severos (Sotelo-Leyva et al. 2020). El cambio de hospedero, de la caña de azúcar al sorgo, puede deberse al cambio de preferencia o al ingreso de un nuevo biotipo de $M$. sacchari (Nibouche et al. 2018). Para su control, los agricultores recurren frecuentemente al uso de insecticidas de origen sintético, como los neonicotinoides, potentes insecticidas sistémicos en el control de insectos chupadores (Tomizawa y Casida 2005). Sin embargo, el uso inadecuado de insecticidas de origen sintético puede afectar negativamente la biodiversidad, la salud de los seres vivos, así como a insectos benéficos (Medrzycki et al. 2003). Considerando estos problemas, es importante la búsqueda de nuevos compuestos insecticidas o sustancias naturales que sean amigables con el medio ambiente y que no resulten nocivos para la salud de los seres vivos.

En este sentido, los insecticidas botánicos pueden ser efectivos para la protección de los cultivos; además, resultan menos costosos, son biodegradables y seguros para el ser humano y el medio ambiente (Silva et al. 2002; Isman 2008).

Una especie vegetal de posible interés es Serjania schiedeana Schltdl (Sapindaceae); conocida como "Cuapalachtli" o "Bejuco de tres costillas", suelen utilizarla los pobladores de la Reserva de la Biosfera de la Sierra de Huautla en Morelos para el ardor de los pies, así como para disminuir el dolor de cintura y espalda, curar heridas y golpes, y para la inflamación de los riñones (Maldonado 1997; Maldonado et al. 2004). En la literatura reciente, se reportan escasas investigaciones sobre la actividad biológica de S. schiedeana; por ejemplo, Sotelo-Leyva et al. (2019) reportan la actividad afidicida de una fracción acuosa de $S$. schiedeana contra $M$. sacchari en dieta artificial, mediante la identificación de las proantocianidinas como compuestos mayoritarios en la fracción acuosa. El efecto insecticida de compuestos de alta y baja polaridad de extractos vegetales ha sido demostrado en áfidos, pero no se reporta la actividad insecticida de extractos de polaridad intermedia S. schiedeana contra ninguna especie de áfido. 
Por lo anterior, el objetivo principal de esta investigación fue evaluar la actividad insecticida en bioensayos de contacto de S. schiedeana contra M. Sacchari, y conocer la composición química de la fracción de acetato de etilo de S. schiedeana por medio de un análisis de cromatografía de gases-masas (CG-MS).

\section{Materiales y MÉtodos}

\section{Colecta y preparación del material vegetal}

Tallos de S. schiedeana (3.7 kg) fueron colectados en septiembre de 2017 en la Reserva de la Biosfera de Huautla, Morelos, México (REBIOSH); se secaron a la sombra durante un mes y se pulverizaron con ayuda de un molino manual. El material seco y pulverizado se maceró con metanol. La extracción se llevó a cabo con ayuda de un rotaevaporador (Laborota ${ }^{\circledR}$ 4000, Heidolph Instruments, Schwabach, Alemania), por tres días por triplicado. El extracto fue fraccionado por un proceso de partición, usando agua y acetato de etilo (1:1). La fracción de acetato de etilo obtuvo un rendimiento de $7.6 \%$; el extracto se congeló a $-20{ }^{\circ} \mathrm{C}$ hasta su uso en los bioensayos.

\section{Insectos}

Se colectaron pulgones en cultivos de sorgo en la comunidad de Tenango, Morelos, y se efectuaron preparaciones permanentes para su identificación mediante las claves taxonómicas de Blackman y Eastop (2000), con la ayuda de Adriana Gabriela Trejo Loyo (Centro de Investigaciones Biológicas, Universidad Autónoma del Estado de Morelos). Los áfidos identificados como Melanaphis sacchari fueron establecidos en plantas sanas de sorgo híbrido, variedad M550@ (Majestic Seeds Co., Hodges, Carolina del Sur, EUA), de $40 \mathrm{~cm}$ de altura, en macetas de plástico, a una temperatura de $24 \pm 2{ }^{\circ} \mathrm{C}$, con $60 \%$ de humedad relativa y un ciclo de luz: oscuridad 12: 12, para la reproducción en el invernadero del Centro de Investigación en Biodiversidad y Conservación de la Universidad Autónoma del Estado de Morelos. A estas plantas de sorgo se les proporcionó riego durante su crecimiento, para mantener su turgencia. Los áfidos fueron suministrados con nuevas plantas de sorgo según fue necesario para mantener la cría.

\section{Bioensayos}

Para cada tratamiento, se colocó una sección de $5 \times 5 \mathrm{~cm}$ de una hoja de sorgo, variedad M550, dentro de una placa de Petri de plástico de $30 \mathrm{~cm}^{3}$, con papel de filtro en el fondo, y se añadió $1 \mathrm{~mL}$ de agua destilada para evitar la deshidratación. Inmediatamente, se colocaron 10 adultos ápteros de $M$. sacchari con un cepillo de pelo de camello. Al extracto previamente obtenido se le agregó Tween® 20 a 0.2\% (Merck KGaA, Darmstadt, Alemania), para permitir que se homogeneizara en agua. Se usó un aerógrafo (Truper ${ }^{\circledR}$ Aero-35, Estado de México, México), para asperjar $0.15 \mathrm{~mL}$ de cada mezcla a concentraciones de 1,000, 2,500, 5,000 y 10,000 ppm sobre los pulgones. Se hicieron dos réplicas, con 5 repeticiones, en un diseño completamente al azar. El porcentaje de mortalidad se registró a las 24,48 y $72 \mathrm{~h}$ después de aplicar los tratamientos. Los insecticidas químicos sintéticos Confial ${ }^{\circledR}$ (ingrediente activo: 1\% de imidacloprid) y Tween ${ }^{\circledR} 20(0.2 \%)$ se usaron como controles.

\section{Cromatografía de gases-masas (CG-MS)}

La fracción de acetato de etilo de los tallos de $S$. schiedeana se analizó con un sistema de cromatografía de gases (Agilent Technology ${ }^{\circledR}$ Wilmington, Carolina del Norte, EUA), acoplado con un espectrómetro de masas, el cual cuenta con detector MSD, una fuente iónica Agilent $5973 \mathrm{~N} \circledast$ a $230{ }^{\circ} \mathrm{C}$ e inyección automática. La espectrometría de masas se llevó a cabo por impacto electrónico. La columna cromatográfica fue una columna HP-5MS® de $30 \mathrm{mx}$ $0250 \mathrm{~mm}$, con un espesor de película de $0.25 \mu \mathrm{m}$. Su temperatura inicial fue de $40{ }^{\circ} \mathrm{C}$, la cual se incrementó a $250{ }^{\circ} \mathrm{C}$ durante $10 \mathrm{~min}$ a una velocidad de $10{ }^{\circ} \mathrm{C}$ min-5, hasta alcanzar $285^{\circ} \mathrm{C}$ durante $20 \mathrm{~min}$. El flujo de gas helio fue de $1 \mathrm{~mL} / \mathrm{min}$, y el tiempo total de ejecución de $20 \mathrm{~min}$. La muestra diluida $(10 \mu \mathrm{L}$ del extracto en $1.0 \mathrm{~mL}$ de solvente) se inyectó ( $2 \mathrm{~mL}$ ) en modo dividido a $250{ }^{\circ} \mathrm{C}$; los espectros se obtuvieron a 1,918 de voltaje de ionización con un rango de 40-450 m/z de masa analizada. Los compuestos en el extracto de $S$. schiedeana se identificaron y autenticaron utilizando sus espectros de masas en comparación con la versión 1.7 A de la biblioteca del National Institute of Standards and Technology (NIST). 


\section{Análisis estadístico}

Los datos de mortalidad se transformaron por la función arcoseno antes de someterse a su análisis estadístico, en un diseño completamente al azar, por medio de un modelo simple, utilizando un procedimiento lineal general. La comparación de medias se efectuó mediante la prueba Tukey, a un nivel de significancia de $P \leq 0.05$. La concentración letal media (CL50) se estimó mediante un análisis Probit. Todos los análisis se hicieron en el programa estadístico SAS ver. 9.0 (SAS 2002).

\section{RESUltados Y Discusión}

\section{Efecto de extractos en $M$. sacchari}

En el Cuadro 1 se muestran los promedios de mortalidad acumulada de los áfidos en función del tiempo de evaluación. El efecto insecticida de los productos aplicados se manifestó desde las $24 \mathrm{~h}$; a este tiempo, el porcentaje mayor se observó para la concentración de 10,000 ppm (24\%). A las 48 h, se observó un aumento en la mortalidad en todos los tratamientos; el mayor efecto se produjo a una concentración de 10,000 ppm (42\%); sin embargo, los mayores porcentajes de mortalidad se observaron a las $72 \mathrm{~h}$; los tratamientos a 10,000 y 5,000 ppm causaron mortalidades de 78 y $46 \%$, respectivamente.

La concentración letal media (CL50) de la fracción de acetato de etilo de tallos de S. schiedeana fue de 6,008 ppm en $72 \mathrm{~h}$. Todos los tratamientos fueron menores y estadísticamente diferentes al control positivo.

En general, los extractos de baja polaridad son efectivos en bioensayos de contacto contra los áfidos, por la afinidad que existe entre su cuerpo graso (Brey et al. 1985) y los compuestos de baja polaridad (ácidos grasos), presentes en las especies vegetales. La mayoría de las investigaciones reportan metodologías por medio de bioensayos de contacto contra áfidos, como el de Ateyyat y Abu-Darwish (2009), quienes evaluaron extractos hexánicos de la corteza de Rhamnus dispermus Ehrenb (Rhamnaceae) contra el pulgón Pterochloroides persicae Cholodkovsky (Hemiptera: Aphididae), con mortalidad de $40 \%$ a 10,000 ppm, en 72 h. En nuestra investigación, la fracción de acetato de etilo de $S$. schiedeana a concentración de 10,000ppm logró una mortalidad mayor (78\%), en el mismo tiempo experimental (72 h). Se evaluó la actividad insecticida por contacto del extracto de éter de petróleo de frutos de Illicium verum Hook. F. (Magnoliaceae), contra Myzus persicae Sulzer (Hemiptera: Aphididae); los resultados revelaron que la mayor mortalidad $(74.4 \%)$ se observó a 1,000 ppm en 72 h (Shi-Guang et al. 2016). En nuestra investigación obtuvimos menores mortalidades en la misma concentración y en mismo tiempo; esto puede ser debido a que los frutos contienen una mayor cantidad de compuestos de baja polaridad, los que podrían ser responsables del mayor efecto insecticida.

Serjania schiedeana es una especie vegetal poco estudiada químicamente. Se ha reportado a las proantocianidinas en una fracción acuosa de tallos de

Cuadro 1. Efecto de la fracción de acetato de etilo de tallos de Serjania schiedeana, en cuatro concentraciones, en adultos ápteros de Melanaphis sacchari, en bioensayos de contacto.

\begin{tabular}{llcc}
\hline \multirow{2}{*}{ Tratamientos (ppm) } & \multicolumn{3}{c}{ Porcentaje de mortalidad $( \pm \mathrm{DE})$} \\
\cline { 2 - 4 } & 24 & \multicolumn{3}{c}{ Horas después de la exposición } \\
\hline 10,000 & $24 \pm 0.5 \mathrm{~b}^{*}$ & $42 \pm 1.2 \mathrm{~b}$ & $78 \pm 1.0 \mathrm{~b}$ \\
5,000 & $4 \pm 0.5 \mathrm{c}$ & $8 \pm 0.4 \mathrm{c}$ & $46 \pm 2.4 \mathrm{~b}$ \\
2,500 & $0 \pm 0 \mathrm{c}$ & $6 \pm 0.5 \mathrm{c}$ & $16 \pm 1.4 \mathrm{c}$ \\
1,000 & $0 \pm 0 \mathrm{c}$ & $12 \pm 0.7 \mathrm{c}$ & $14 \pm 1.0 \mathrm{c}$ \\
Control positivo & $100 \pm 0 \mathrm{a}$ & $100 \pm 0 \mathrm{a}$ & $100 \pm 0 \mathrm{a}$ \\
Control negativo & $0 \pm 0 \mathrm{c}$ & $0 \pm 0 \mathrm{c}$ & $4 \pm 0.5 \mathrm{c}$ \\
\hline
\end{tabular}

*Las medias en cada columna con una letra diferente fueron significativamente diferentes, prueba de comparación múltiple de medias Tukey $(P \leq 0.05)$. (LC50 = 6,008 ppm en $72 \mathrm{~h}$ ). 
S. schiedeana; a éstas se les atribuye una actividad afidicida (Sotelo-Leyva et al. 2019). En esta investigación se reportan por primera vez compuestos de baja e intermedia polaridad de S. schiedeana. El análisis de CG-MS del extracto acetato de etilo de S. schiedeana reveló la presencia de diterpenos y ácidos grasos esterificados (Cuadro 2).
Picrasma crenata Vell. (Simaroubaceae) es tóxico contra Myzus persicae Sulzer (Hemiptera: Aphididae), pues provocó una mortalidad de $100 \%$ en concentraciones de 3,000 y 6,000 ppm. En un estudio de Sotelo-Leyva et al. (2020), se evaluaron distintos extractos de diversas partes de Ricinus communis L. (Euphorbiaceae), contra M. sacchari (Hemiptera: Aphididae);

Cuadro 2. Composición química de la fracción de acetato de etilo de Serjania schiedeana.

\begin{tabular}{llll}
\hline Constituyentes & $\begin{array}{l}\text { Tiempo de } \\
\text { retención } \\
(\text { min) }\end{array}$ & $\begin{array}{l}\text { Cantidad } \\
(\%)\end{array}$ & $\begin{array}{l}\text { Fórmula } \\
\text { molecular }\end{array}$ \\
\hline Fitol & 17.72 & 1.18 & $\mathrm{C}_{20} \mathrm{H}_{40} \mathrm{O}$ \\
Fitona & 17.79 & 1.13 & $\mathrm{C}_{18} \mathrm{H}_{36} \mathrm{O}$ \\
Palmitato de metilo & 18.61 & 38.66 & ${\mathrm{C} 1{ }_{7} \mathrm{H}_{34} \mathrm{O}_{2}}$ \\
Araquinato de metilo & 19.57 & 1.05 & $\mathrm{C}_{21} \mathrm{H}_{42} \mathrm{O}_{2}$ \\
4,8,12,16-tetrametilheptadecan-4-olido & 20.24 & 15.99 & $\mathrm{C}_{21} \mathrm{H}_{40} \mathrm{O}_{2}$ \\
Linoleato de metilo & 20.30 & 17.17 & $\mathrm{C}_{19} \mathrm{H}_{34} \mathrm{O}_{2}$ \\
Oleato de metilo & 20.51 & 3.41 & $\mathrm{C}_{19} \mathrm{H}_{36} \mathrm{O}_{2}$ \\
Estearato de metilo & 22.27 & 1.23 & $\mathrm{C}_{19} \mathrm{H}_{38} \mathrm{O}_{2}$ \\
Behenato de metilo & 22.58 & 2.07 & $\mathrm{C}_{23} \mathrm{H}_{46} \mathrm{O}_{2}$ \\
Tetracosanoato de metilo & 24.39 & 12.97 & $\mathrm{C}_{25} \mathrm{H}_{50} \mathrm{O}_{2}$ \\
(4- (2,6-di-terc-butil-4-metoxifenoxi) -3-nitro-4-oxobutil) & 29.45 & 4.13 & $\mathrm{C}_{28} \mathrm{H}_{44} \mathrm{~N}_{2} \mathrm{O}_{7}$ \\
prolinato de terc-butilo & & & \\
Total & & & \\
\hline
\end{tabular}

La actividad insecticida de extractos no polares contra áfidos ya ha sido comprobada; por ejemplo, Singh et al. (1988) determinaron la actividad insecticida por contacto de los extractos acuosos, etanólicos y hexánicos de Azadirachta indica A. Juss (Meliaceae) contra Lipaphis erysimi Kalt. (Hemiptera: Aphididae); el extracto no polar (hexánico) mostró una mayor actividad insecticida (48\%) a una concentración de $5,000 \mathrm{ppm}$. Nuestro estudio reveló una mortalidad similar (46\%) a 5,000 ppm, en $72 \mathrm{~h}$. Cheikh-Affene et al. (2012) evaluaron extractos de n-hexano de especies de rosas, contra Aphis fabae Scop. y Acyrthosiphon pisum Scop. (Hemiptera: Aphididae). Rosa dumetorum Thuillier (Rosaceae) presentó el mayor porcentaje de mortalidad para ambos áfidos, y mediante un análisis de CG-MS se identificó al ácido araquidónico como componente principal. Rodríguez et al. (2012) reportaron que el extracto de acetato de etilo de el mayor efecto insecticida lo presentó el extracto de $n$-hexano de las hojas (96\%); se identificó una mezcla de dos ácidos grasos (mirístico y esteárico) como componentes principales en la fracción activa.

El palmitato de metilo fue el compuesto mayoritario en la fracción de acetato de etilo de S. schiedeana (38.66\%); este compuesto ha sido mencionado con actividad acaricida (Wang et al. 2010); sin embargo, no existen reportes sobre el efecto biológico del palmitato de metilo en áfidos.

\section{CONCLUSIONES}

La fracción de acetato de etilo de $S$. schiedeana mostró actividad insecticida contra $M$. sacchari. Los compuestos de baja polaridad como el palmitato de metilo presente en S. schiedeana podrían estar involucrados en el efecto insecticida del extracto. 


\section{AGRADECIMIENTOS}

Este trabajo fue financiado por PRODEP 511-6/17-6413/

UAGro-219. Agradecemos al Centro de Investigación

Biomédica del Sur (CIBIS-IMSS), por el apoyo técnico. 


\section{LITERATURA CITADA}

Ateyyat MA, Abu-Darwish MS. 2009. Short communication. Insecticidal activity of different extracts of Rhamnus dispermus (Rhamnaceae) against peach trunk aphid, Pterochloroides persicae (Homoptera: Lachnidae). Spanish Journal of Agricultural Research 7: 160-164.

Blackman RL, Eastop VF. 2000. Aphids on the World's Crops: an Identification and Information Guide. John Wiley \& Sons. Chichester, U.K.

Brey TP, Ohayon H, Lesourd M, Castex H, Roucachet J, Latge JP. 1985. Ultrastructure and chemical composition of the outer layers of the cuticle of the pea aphid Acyrthosiphon pisum (Harris). Comparative Biochemistry and Physiology. Part A: Physiology 82: 401-411. http://doi.org/10.1016/0300-9629(85)90875-8

Cheikh-Affene B, Chaieb I, Haouala F, Harzallah-Skhiri F. 2012. Effects of wild rose (Rosa sp.) crude seed extracts on two legume aphids. Bulletin of University of Agricultural Sciences and Veterinary Medicine. Horticulture 1: 9-15.

Chuck-Hernández C, Pérez-Carrillo E, Heredia-Olea E, Serna-Saldívar SO. 2011. Sorgo como un cultivo multifacético para la producción de bioetanol en México: tecnologías, avances y áreas de oportunidad. Revista Mexicana de Ingeniería Química 10: 529-549.

[FIRA] Fideicomisos Instituidos en Relación con la Agricultura. [internet]. 2016. Panorama agroalimentario. Sorgo. [cited 2019 Dic]. Disponible en: https:// www.gob.mx/cms/uploads/attachment/file/200640/ Panorama_Agroalimentario_Sorgo_2016.pdf.

Isman MB. 2008. Botanical insecticides: for richer, for poorer. Pest Management Science 64: 8-11. http://doi. org/10.1002/ps.1470

Maldonado AB. 1997. Aprovechamiento de los recursos florísticos de la Sierra de Huautla, Morelos. Tesis de Maestría en Ciencias. Universidad Nacional Autónoma de México. Ciudad de México, México.

Maldonado B, Ortiz A, Dorado O. 2004. Preparados galénicos e imágenes de plantas medicinales. Una alternativa para promotoras de la salud en la Reserva de la Biosfera Sierra de Huautla. Centro de Educación Ambiental e Investigación Sierra de Huautla. Morelos, México.

Medrzycki P, Montanari R, Bortolotti L, Sabatini AG, Maini S, Porrini C. 2003. Effects of imidacloprid administered in sub-lethal doses on honey bee behaviour. Laboratory tests. Bulletin of Insectology 56: 59-62.
Nibouche S, Costet L, Holt JR, Jacobson A, Pekarcik A, Sadeyen J, Armstrong JS, Peterson GC, McLaren N, Medina RF. 2018. Invasion of sorghum in the Americas by a new sugarcane aphid (Melanaphis sacchari) superclone. PLoS ONE 13: e0196124. https://doi.org/10.1371/ journal.pone.0196124

Rodríguez S, Regonat M, Carrizo P, Meilan J, Wagner M, Gurni A. 2012. Activity of nonpolar extracts from Picrasma crenata (Simaroubaceae) against Myzus persicae (Hemiptera: Aphididae). Revista Latinoamericana de Química 3: 113-120.

[SAS] Statistical Analysis System. 2002. SAS. System for Windows, Versión 9.0. Institute. Cary, USA.

Schenck S, Lehrer AT. 2007. Factors affecting the transmission and spread of sugarcane yellow leaf virus. Plant Disease 84: 1085-1088. https://doi.org/10.1094/ PDIS.2000.84.10.1085

Shi-Guang L, Ben-Guo Z, Mao-Ye L, Su L, Ri-Mao H, HuaFeng L. 2016. Chemical composition of Illicium verum fruit extract and its bioactivity against the peach-potato aphid, Myzus persicae (Sulzer). Arthropod-Plant Interactions 11: 203-212. https://doi.org/10.1007/s11829-016-9480-6

[SIAP] Servicio de Información Agroalimentaria y Pesquera. [internet]. 2019. Avance de siembras y cosechas resumen nacional por estado. [cited 2019 Dic]. Disponible en: http://www.siap.gob.mx/ cierre-de-la-produccion-agricola-por-cultivo/.

Silva G, Lagunes A, Rodríguez JC, Rodríguez D. 2002. Insecticidas vegetales: una vieja y nueva alternativa para el manejo de plagas. Manejo Integrado de Plagas y Agroecología 66: 4-12.

Singh RP, Devakumar C, Dhingra S. 1988. Activity of neem (Azadirachta indica A. Juss) seed kernel extracts against the mustard aphid, Lipaphis erysimi. Phytoparasitica 16: 225-230. https://doi.org/10.1007/BF02979523

Sotelo-Leyva C, Salinas-Sánchez DO, Rivas-González JM, Dorado O, Arias DM, González-Cortazar M, Zamilpa A. 2019. Aphidicidal activity of an aqueous fraction of Serjania schiedeana against Melanaphis sacchari. Southwestern Entomologist. 44: 585-594. https://doi. org/10.3958/059.044.0304

Sotelo-Leyva C, Salinas-Sánchez DO, Peña-Chora G, Trejo-Loyo AG, González-Cortázar M, Zamilpa A. 2020. Insecticidal compounds in Ricinus communis L. (Euphorbiaceae) to control Melanaphis sacchari Zehntner (Hemiptera: Aphididae). Florida Entomologist 103: 9195. https://doi.org/10.1653/024.103.0415 
Tomizawa M, Casida JE. 2005. Neonicotinoid insecticide toxicology: Mechanisms of selective action. Annual Review Pharmacology and Toxicology 45: 247-68. $\quad$ https://doi.org/10.1146/annurev. pharmtox.45.120403.095930

Wang YN, Wang XH, Jin YS, Bu CY, Cheng J, Zhao LL, Shi GL. 2010. Assessment of the contact toxicity of methyl palmitate on Tetranychus viennensis (Acari: Tetranychidae). Journal of Economic Entomology 14: 1372-1377. https://doi.org/10.1603/ec09128 